International Journal of Pure and Applied Mathematics

Volume 93 No. 6 2014, 799-802

ISSN: 1311-8080 (printed version); ISSN: 1314-3395 (on-line version)

url: http://www.ijpam.eu

doi: http://dx.doi.org/10.12732/ijpam.v93i6.5

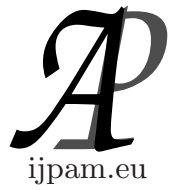

\title{
ON THE STRATIFICATION BY X-RANKS OF A PROJECTIVE SPACE (MAXIMAL AND SUBMAXIMAL X-RANKS)
}

\author{
E. Ballico \\ Department of Mathematics \\ University of Trento \\ 38123 Povo (Trento) - Via Sommarive, 14, ITALY
}

\begin{abstract}
Let $X \subset \mathbb{P}^{N}$ be an integral and non-degenerate variety. For each $P \in \mathbb{P}^{N}$ the X-rank $r_{X}(P)$ of $P$ is the minimal cardinality of a subset of $X$ whose linear span contains $P$. For each $x>0$ set $A_{X}(x):=\left\{P \in \mathbb{P}^{N}: r_{X}(P)=x\right\}$. We prove that if $r_{\max }$ is the maximum of all $r_{X}(P)$ and $a:=\operatorname{dim}(X) \leq N-2$, then $\operatorname{dim}\left(A_{X}\left(r_{\max }-1\right)\right) \geq \max \left\{1+a, \operatorname{dim}\left(A_{X}\left(r_{\max }\right)\right)+2\right\}$.
\end{abstract}

AMS Subject Classification: 14N05, 15A69

Key Words: X-rank, open rank, secant variety

\section{Introduction}

Let $X \subset \mathbb{P}^{N}$ be an integral and non-degenerate variety. For each $P \in \mathbb{P}^{N}$ the $X$-rank $r_{X}(P)$ of $P$ is the minimal cardinality of a finite set $S \subset X$ such that $P \in\langle S\rangle$, where \langle\rangle denote the linear span. The open $X$-rank $w_{X}(P)$ of $P$ is the minimal integer $t$ with the following property. Fix a closed proper subset $B \varsubsetneqq X$. Then there is a set $S \subset X \backslash B$ such that $P \in\langle S\rangle$ and $\sharp(S)=t([4])$. The open X-rank $w_{X}(P)$ measures the following game. You fix $P$. Then your opponent fix $B \varsubsetneqq X$. Then you need to describe $P$ only using points of $X \backslash B$. Call $r_{\max }$ (resp. $w_{\max }$ ) the maximum of the integers $r_{X}(P)$ (resp. $w_{X}(P)$ ), $P \in \mathbb{P}^{N}$. In many cases the X-rank is important for real life applications (e.g. if $X$ is a Veronese variety for the decomposition of a homogeneous polynomial

Received: February 25, 2014

(c) 2014 Academic Publications, Ltd. url: www.acadpubl.eu 
into a sum of power of linear forms and if $X$ is a Segre embedding for the tensor decomposition of a tensor into rank one tensors) ([3], [5]). Hence it is useful to have upper bounds for the integer $r_{\max }$. For every $P$ we have $w_{X}(P) \geq r_{X}(P)$. There is a non-empty open subset $U$ of $\mathbb{P}^{N}$ such that $r_{X}(P)=r_{X}(Q)$ for all $P, Q \in U$. The integer $r_{X}(P), P \in U$, is called the generic $X$-rank $r_{\text {gen }}$. Using that the image of a constructible subset by an algebraic map is constructible (Chevalley's theorem), one can also show the existence of a non-empty open subset $W$ of $\mathbb{P}^{N}$ such that $w_{X}(P)=w_{X}(Q)$ for all $P, Q \in W$. We call this integer $w_{\max }:=w_{X}(P), P \in W$, the generic open $X$-rank. It is well-know that the integer $r_{\max }$ is computed in the following way. For each integer $b \geq 1$ let $\sigma_{b}(X)$ denote the closure of the union of all linear spaces $\langle S\rangle$ for all $S \subset X$ with $\sharp(S)=b$. The variety $\sigma_{b}(X)$ is called the $b$-secant variety of $X$. The integer $r_{\text {gen }}$ is the minimal integer $b$ such that $\sigma_{b}(X)=\mathbb{P}^{N}$. In many cases (e.g. if $X$ is the Veronese variety) this observation gives an explicit solution for the integer $r_{\text {gen }}$. Recently G. Blekerherman and Z. Teitler proved that $r_{\max } \leq 2 r_{\text {gen }}$ ([2, Theorem $1]$ ). In some cases they also give a better bound under stronger assumptions and studied the corresponding concept over the field $\mathbb{R}([2$, Theorem 3$])$. We observe that the proof of $[2$, Theorem 1] works also for the open X-rank (see Proposition 1). For each integer $x>0$ set $A_{X}(x):=\left\{P \in \mathbb{P}^{N}: r_{X}(P)=x\right\}, A_{X}(\geq x):=$ $\left\{P \in \mathbb{P}^{N}: r_{X}(P) \geq x\right\}$ and $A_{X}(\leq x):=\left\{P \in \mathbb{P}^{N}: r_{X}(P) \leq x\right\}$. By Chevalley's theorem the sets $A_{X}(x), A_{x}(\leq x)$ and $A_{X}(\geq x)$ are locally constructible. For any two irreducible subsets $A, B$ of $\mathbb{P}^{N}$ the join $J(A, B) \subseteq \mathbb{P}^{N}$ is the closure in $\mathbb{P}^{N}$ of the union of all lines $\langle\{O, P\}\rangle$ with $O \in A, P \in B$ and $O \neq P$, with the convention $J(A, B):=A$ if $A$ and $B$ are just points and $A=B$. For any subset $E \subseteq \mathbb{P}^{N}$ let $\bar{E}$ denote the closure of $E$ in $\mathbb{P}^{N}$.

In this note we prove the following result.

Theorem 1. Fix an integer $x \geq 2$ and fix any irreducible algebraic set $T \subseteq A_{X}(x)$ such that $\bar{T}$ is an irreducible component of $\overline{A_{X}(x)}$. Then there is an irreducible subset $E \subset A_{X}(x-1) \cup A_{X}(\geq x+1)$ such $J(X, \bar{T}) \subseteq \bar{E}$. We have $\operatorname{dim}(E) \geq \max \{\operatorname{dim}(T)+2, \operatorname{dim}(X)+1\}$.

Theorem 1 is more interesting in the range $x \geq 2+w_{\text {gen }}$, because $J\left(X, \sigma_{b}(X)\right)=$ $\sigma_{b+1}(X)$ for all $b>0$. Notice that $A_{X}\left(\geq r_{\max }+1\right)=\emptyset$. Hence Theorem 1 gives the following corollary.

Corollary 1. Assume $X \neq \mathbb{P}^{N}$. Then $\operatorname{dim}\left(A_{X}\left(r_{\max }-1\right)\right) \geq 1+\operatorname{dim}(X)$ and $\operatorname{dim}\left(A_{X}\left(r_{\max }-1\right)\right) \geq \min \left\{N, \operatorname{dim}\left(A_{X}\left(r_{\max }\right)+2\right\}\right.$.

In particular both the integers $r_{\max }$ and $r_{\max }-1$ are the X-ranks of some points of $\mathbb{P}^{N}$.

We work over an algebraically closed field $\mathbb{K}$. 


\section{Proofs and remarks}

Proposition 1. We have $w_{\max } \leq 2 w_{\max }$.

Proof. Let $W \subset \mathbb{P}^{N}$ be any nonempty open subset such that $w_{X}(Q)=w_{\text {gen }}$ for all $Q \in W$. Fix $P \in \mathbb{P}^{N}$ and a closed subset $B \varsubsetneqq X$. Let $L \subset \mathbb{P}^{N}$ be a line through $P$ and intersecting $W$. Since $W \cap L$ is non-empty and open in $L$, there are $P_{1}, P_{2} \in W \cap L$ with $P_{1} \neq P_{2}$. By the definition of the integer $w_{X}\left(P_{i}\right), i=1,2$, there is $S_{i} \subset X \backslash B$ such that $P_{i} \in\left\langle S_{i}\right\rangle$ and $\sharp\left(S_{i}\right) \leq w_{\text {gen }}$. We have $P \in\left\langle S_{1} \cup S_{2}\right\rangle, S_{1} \cup S_{2} \subset X \backslash B$ and $\sharp\left(S_{1} \cup S_{2}\right) \leq 2 w_{\text {gen. }}$. Hence $w_{X}(Q) \leq 2 w_{\text {gen }}$.

We may improve Proposition 1 in the following way ([2, Theorem 3]).

Proposition 2. Fix an integer $\rho<w_{\text {gen }}$. Assume the existence of a closed irreducible hypersurface $T \subset \mathbb{P}^{N}$ and a non-empty open subset $U \subset T$ such that $w_{X}(P) \leq \rho$ for all $P \in U$. Then $w_{X}(Q) \leq w_{\text {gen }}+\rho$ for all $Q \in \mathbb{P}^{N} \backslash T$.

Proof. Fix $Q \in \mathbb{P}^{N} \backslash T$ and a closed subset $B \varsubsetneqq X$. Let $W \subset \mathbb{P}^{N}$ a nonempty open subset such that $w_{X}(P)=w_{\text {gen }}$ for all $P \in W$. Let $L$ be a general line through $Q$. Since $T$ is a hypersurface, we have $L \cap T \neq \emptyset$. Since $U$ is open and dense in $T$, for a general $L$ we have $L \cap U \neq \emptyset$. Since $T \subseteq \mathbb{P}^{N} \backslash W$, we have $P_{1} \neq P_{2}$. Take $S_{i} \subset X \backslash B, i=1,2$, such that $P_{i} \in\left\langle S_{i}\right\rangle, \sharp\left(S_{1}\right) \leq w_{\text {gen }}$ and $\sharp\left(S_{2}\right) \leq \rho$. Since $\sharp\left(S_{1} \cup S_{2}\right) \leq \rho+w_{\text {gen }}$, we have $w_{X}(Q) \leq w_{\text {gen }}+\rho$.

Proposition 3. Fix an integer $\rho<w_{\text {gen }}$. Assume the existence of a closed irreducible hypersurface $T \subset \mathbb{P}^{N}$ and a non-empty open subset $U \subset T$ such that $w_{X}(P) \leq \rho$ for all $P \in U$ and $d:=\operatorname{deg}(T) \geq 2$. Fix $Q \in \mathbb{P}^{N} \subset T$. If $\operatorname{char}(\mathbb{K})>0$ assume that $T$ is not a strange variety with $Q$ as one of its strange points. Then $w_{X}(Q) \leq 2 \rho$.

Proof. Let $\ell: \mathbb{P}^{N} \backslash\{Q\} \rightarrow \mathbb{P}^{N-1}$ denote the linear projection. Since $Q \notin T$, $\ell \mid T$ is a morphism. The morphism $\ell \mid T$ is a finite morphism of degree $d$. Since $Q$ is not a strange point of $T, \ell \mid T$ is separable. Hence for a general line $L$ through $P$ the scheme $T \cap L$ is formed by $d$ distinct points, all of them contained in $U$. Taking $P_{1}, P_{2} \in L \cap U$ with $P_{1} \neq P_{2}$ we get $w_{X}(Q) \leq 2 \rho$.

Remark 1. Assume $p:=\operatorname{char}(\mathbb{K})>0$. Let $T \subset \mathbb{P}^{N}$ be an integral hypersurface of degree $d \geq 2$. Fix $Q \in \mathbb{P}^{N} \backslash T$. If $T$ is strange, then $p \mid d$. The proof of Proposition 3 gives $w_{X}(Q) \leq 2 \rho$ if $\ell \mid T$ is not purely inseparable. If $\ell \mid T$ is purely inseparable, then $d$ is a $p$-power. 
Proof of Theorem 1: Fix a general $Q \in T$. Since $x \geq 2$, we have $Q \notin X$. Fix a general $O \in X$ and fix any $P \in\langle\{O, Q\}\rangle \backslash\{O\}$. Since $Q \in\langle\{P, O\}\rangle$ (even in the case $P=Q$ ), we have $r_{X}(Q) \leq 1+r_{X}(P)$. Hence a general element of the irreducible variety $J(X, T)$ has X-rank at least $x-1$. Since $X$ is non-degenerate and $\bar{T} \neq \mathbb{P}^{N}$, a a general $O \in X$ is not a vertex of $\bar{T}$. Hence $\operatorname{dim}(J(\{O\}, \bar{T})=\operatorname{dim}(T)+1$ ([1, part (i) of Proposition 1.3]). If $b:=\operatorname{dim}(T)=N-1$, then we are done. Now assume $b \leq N-2$. Since $X$ is non-degenerate, the variety $J(\{O\}, \bar{T})$ is not a cone with vertex containing $X$. Hence $\operatorname{dim}(J(X, \bar{T})) \geq b+2([1$, part (ii) of Proposition 1.3]). Now we reverse the role of $T$ and $X$. Fix a general $Q \in T$. Since $r_{X}(Q)=x>1$, we have $Q \notin X$. Hence $\operatorname{dim}(J(T, X)) \geq \operatorname{dim}(J(\{Q\}, X))=\operatorname{dim}(X)+1$.

Assume $a:=\operatorname{dim}(X) \leq N-2$ and $b:=\operatorname{dim}(T) \geq 2$. To get $\operatorname{dim}(J(X, \bar{T})) \geq$ $\operatorname{dim}(X)+2$ it would be sufficient to assume that for a general $O \in T$ the cone $J(\{O\}, X)$ has not a vertex containing $T$. We know no natural condition which assures it.

\section{Acknowledgements}

The author was partially supported by MIUR and GNSAGA of INdAM (Italy).

\section{References}

[1] B. Ålandsvik, Joins and higher secant varieties, Math. Scand. 61 (1987), 213-222.

[2] G. Blekherman and Z. Teitler, On maximum, typical, and generic ranks, arXiv:1402.2371.

[3] A. Iarrobino, V. Kanev, Power sums, Gorenstein algebras, and determinantal loci, Appendix C by Iarrobino and Steven L. Kleiman, Lecture Notes in Mathematics, 1721. Springer-Verlag, Berlin, 1999.

[4] J. Jelisiejew, An upper bound for the Waring rank of a form, arXiv:1305.6957.

[5] J. M. Landsberg, Tensors: Geometry and Applications Graduate Studies in Mathematics, Vol. 128, Amer. Math. Soc. Providence, 2012. 